\title{
CAPACITY OF MULTIPLE ACCESS CHANNELS WITH CORRELATED JAMMING*
}

\author{
Shabnam Shafiee and Sennur Ulukus \\ Department of Electrical and Computer Engineering \\ University of Maryland \\ College Park, MD
}

\begin{abstract}
We investigate the behavior of two users and one jammer in an $A W G N$ channel with and without fading when they participate in a non-cooperative zero-sum game, with the channel's input/output mutual information as the objective function. We assume that the jammer can eavesdrop the channel and can use the information obtained to perform correlated jamming. Under various assumptions on the channel characteristics, and the extent of information available at the users and the jammer, we show the existence, or otherwise nonexistence of a simultaneously optimal set of strategies for the users and the jammer. Whenever the game has a solution, we find the corresponding user and jammer strategies, and whenever the game does not admit a solution, we find the max-min user strategies and the corresponding jammer strategy.
\end{abstract}

\section{INTRODUCTION}

Correlated jamming, the situation where the jammer has full or partial knowledge about the user signals has been studied in the information-theoretic context under various assumptions [1-3]. In [1] the best transmitter/jammer strategies are found for a single user AWGN channel with a jammer who has full or partial knowledge of the transmitted signal. In [2], the problem is extended to a single user MIMO fading channel. This model has been further extended in [3], to consider fading in the channel between the jammer and the receiver. In [3] various assumptions are made on the availability of the user channel state at the user, and the jammer channel state at the jammer.

In this paper, we study a multi-user system under correlated jamming. Partial results of this study have been reported in [4]. We consider a system of two users

\footnotetext{
${ }^{*}$ This work was supported by NSF Grants ANI 02-05330 and CCR 03-11311; and ARL/CTA Grant DAAD 19-01-2-0011.
}

and one jammer who has full or partial knowledge of the user signals through eavesdropping. In the nonfading two user channel, we show that the game has a solution which is Gaussian signalling for the users, and linear jamming for the jammer. Here we define linear jamming as employing a linear combination of the available information about the user signals plus Gaussian noise. We then consider fading in the user channels. As opposed to [3], where the user channel states could only be known at the users, we assume the possibility of the jammer gaining information about the user channel states by eavesdropping the feedback channel from the receiver to the users and show that if the jammer is not aware of the user channel states, it would disregard its eavesdropping information and only transmit Gaussian noise. If the jammer knows the user channel states but not the user signals, the game has a solution which is composed of the optimal user and jammer power allocation strategies over the channel states, together with Gaussian signalling and linear jamming at each channel state. If the jammer knows the user channel states and the user signals, the game does not always have a Nash equilibrium solution, in which case, we characterize the max-min user strategies, and the corresponding jammer best response.

The term capacity will hereafter always refer to the channel's information capacity, defined as the channel's maximum input/output mutual information [5].

\section{SYSTEM MODEL}

Figure 1 shows a communication system with two users and one jammer. In the absence of fading, the attenuations of the user channels are known to everyone. The AWGN channel with two users and one jammer is

$$
Y=\sqrt{h_{1}} X_{1}+\sqrt{h_{2}} X_{2}+\sqrt{\gamma} J+N
$$




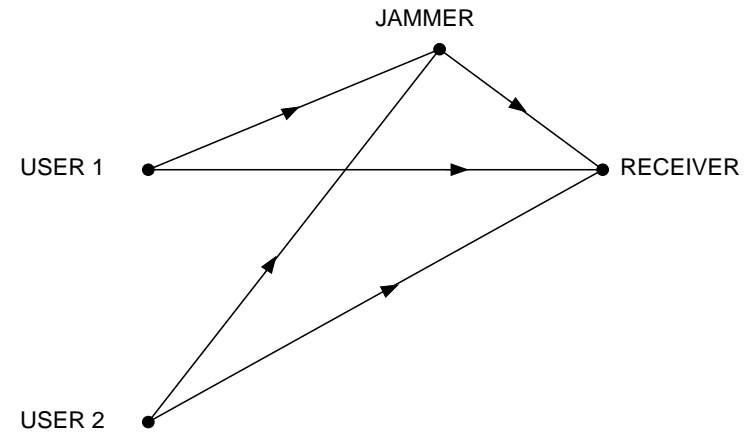

Figure 1: A communication system with two users and one jammer.

where $X_{i}$ is the the $i^{\text {th }}$ user's signal, $h_{i}$ is the attenuation of the $i^{t h}$ user's channel, $J$ is the jammer's signal, $\gamma$ is the attenuation of the jammer's channel and $N$ is a zeromean Gaussian random variable with variance $\sigma_{N}^{2}$. To model fading in the received powers, we consider $h_{i}$ as fading random variables, and to further model the phase of the user channel coefficients, we substitute the scalar attenuations $\sqrt{h_{i}}$ with complex fading random variables $H_{i}, i=1,2$. The power constraints are

$$
\begin{aligned}
& E\left[X_{i}^{2}\right] \leq P_{i}, \quad i=1,2 \\
& E\left[J^{2}\right] \leq P_{J}
\end{aligned}
$$

We analyze both cases when the jammer has perfect information about the user signals, and imperfect information gained through eavesdropping, where the jammer's eavesdropping link is also a multi-user AWGN channel

$$
Y_{e}=\sqrt{g_{1}} X_{1}+\sqrt{g_{2}} X_{2}+N_{e}
$$

where $g_{i}$ is the attenuation of the $i^{\text {th }}$ user's eavesdropping channel, $i=1,2$, and $N_{e}$ is a zero-mean Gaussian random variable with variance $\sigma_{e}^{2}$.

\section{JAMMING WITH COMPLETE INFORMATION}

In this section, we consider the two user non-fading channel in (1), and assume that the jammer knows the user signals. The jammer and the two users are involved in a zero-sum game with the input/output mutual information as the objective function. We investigate the existence and uniqueness of a Nash equilibrium solution for this game [6]. A Nash equilibrium is a set of strategies, one for each player, such that no player has an incentive for unilaterally changing its own strategy.

For $\left(X_{1}, X_{2}, Y\right) \sim f\left(x_{1}\right) f\left(x_{2}\right) f\left(y \mid x_{1}, x_{2}\right)$, the in- put/output mutual information $I\left(X_{1}, X_{2} ; Y\right)$ is a concave function of $f\left(x_{1}\right)$ for fixed $f\left(x_{2}\right)$ and $f\left(y \mid x_{1}, x_{2}\right)$, a concave function of $f\left(x_{2}\right)$ for fixed $f\left(x_{1}\right)$ and $f\left(y \mid x_{1}, x_{2}\right)$, and a convex function of $f\left(y \mid x_{1}, x_{2}\right)$ for fixed $f\left(x_{1}\right)$ and $f\left(x_{2}\right)$, therefore, $I\left(X_{1}, X_{2} ; Y\right)$ has a saddle point, which is the Nash equilibrium solution of the game [4]. In the sequel, we show that when the users employ Gaussian signalling, the best jamming strategy is linear jamming, and when the jammer employs linear jamming, the best strategy for the users is Gaussian signalling, which proves that this set of strategies is a Nash equilibrium solution for the game.

First assume that the jammer employs linear jamming

$$
J=\rho_{1} X_{1}+\rho_{2} X_{2}+N_{J}
$$

Using (1), the output of the channel will be

$$
\begin{aligned}
Y= & \left(\sqrt{h_{1}}+\sqrt{\gamma} \rho_{1}\right) X_{1}+\left(\sqrt{h_{2}}+\sqrt{\gamma} \rho_{2}\right) X_{2} \\
& +\sqrt{\gamma} N_{J}+N
\end{aligned}
$$

which describes an AWGN multiple access channel, for which the best signalling for the users is Gaussian [5].

Next, we should show that if the users perform Gaussian signalling, then the best jamming strategy is linear jamming. The channel output is as in (1) with the input/output mutual information

$$
I\left(Y ; X_{1}, X_{2}\right)=h\left(X_{1}, X_{2}\right)-h\left(X_{1}, X_{2} \mid Y\right)
$$

The jammer can only affect $h\left(X_{1}, X_{2} \mid Y\right)$

$$
\begin{aligned}
h\left(X_{1}, X_{2} \mid Y\right) & =h\left(X_{1}-a_{1} Y, X_{2}-a_{2} Y \mid Y\right) \\
& \leq h\left(X_{1}-a_{1} Y, X_{2}-a_{2} Y\right) \\
& \leq \frac{1}{2} \log \left((2 \pi e)^{2}|\boldsymbol{\Lambda}|\right)
\end{aligned}
$$

where $\boldsymbol{\Lambda}$ is the covariance matrix of $\left(X_{1}-a_{1} Y, X_{2}-\right.$ $\left.a_{2} Y\right)$. We choose $a_{1}=E\left[X_{1} Y\right] / E\left[Y^{2}\right]$ and $a_{2}=$ $E\left[X_{2} Y\right] / E\left[Y^{2}\right]$ and prove the optimality of linear jamming in two steps. First, we consider the set of all jamming signals which result in the same $\boldsymbol{\Lambda}$ value, and show that if this set includes a linear jammer, then that linear jammer is optimal over this set. Then, we consider the set of all feasible jamming signals and show that for any jamming signal in this set, there exists a linear jammer in this set resulting in the same $\boldsymbol{\Lambda}$ value. The feasibility is in the sense of the jammer's available power.

Consider the set of all jamming signals which result in the same $\boldsymbol{\Lambda}$ value in (10). Assume that there is a linear jamming signal in this set. For this jamming sig- 
nal, $X_{1}-a_{1} Y, X_{2}-a_{2} Y$ and $Y$ are jointly Gaussian, and $X_{1}-a_{1} Y$ and $X_{2}-a_{2} Y$ are uncorrelated with and consequently, independent of $Y$. We conclude that this jamming signal achieves both (9) and (10) with equality and is the optimal jamming strategy over this set.

Now we show that any $\boldsymbol{\Lambda}$ achievable by any feasible jammer, is also achievable by a feasible linear jammer. For the chosen values of $a_{1}$ and $a_{2}, \boldsymbol{\Lambda}$ can be expressed as a function of $E\left[X_{1} J\right]$ and $E\left[X_{2} J\right]$ [4]. Consider any jamming signal $J$. Define $R$ as

$$
R=J-X_{1} \frac{E\left[X_{1} J\right]}{P_{1}}-X_{2} \frac{E\left[X_{2} J\right]}{P_{2}}
$$

Note that $R$ is uncorrelated with $X_{1}$ and $X_{2}$. The power of this jamming signal is

$$
E\left[J^{2}\right]=\frac{E\left[X_{1} J\right]^{2}}{P_{1}}+\frac{E\left[X_{2} J\right]^{2}}{P_{2}}+E\left[R^{2}\right]
$$

For this jamming signal to be feasible, we should have

$$
\frac{E\left[X_{1} J\right]^{2}}{P_{1}}+\frac{E\left[X_{2} J\right]^{2}}{P_{2}} \leq P_{J}
$$

Now define a linear jamming signal as in (5), where

$$
\rho_{i}=\frac{E\left[X_{i} J\right]}{P_{i}}, i=1,2
$$

and $N_{J}$ is an independent Gaussian random variable with power $E\left[R^{2}\right]$. This linear jammer has the same power as $J$ and therefore is feasible. Moreover, it results in the same $|\boldsymbol{\Lambda}|$ value as $J$. This means that, there exists a feasible linear jamming signal which is as effective as any other feasible jamming signal, and this concludes the proof.

The next step is to find the jamming coefficients $\rho_{1}$ and $\rho_{2}$ that minimize the mutual information in (7). Minimizing the mutual information here is equivalent to minimizing the SNR [4]

$$
S N R=\frac{\left(\sqrt{h_{1}}+\sqrt{\gamma} \rho_{1}\right)^{2} P_{1}+\left(\sqrt{h_{2}}+\sqrt{\gamma} \rho_{2}\right)^{2} P_{2}}{\gamma \sigma_{N_{J}}^{2}+\sigma_{N}^{2}}
$$

The Karush-Kuhn-Tucker (KKT) necessary conditions result in [4]

$$
\left(\rho_{1}, \rho_{2}\right)=\left\{\begin{array}{lll}
\left(-\frac{\sqrt{h_{1}}}{\sqrt{\gamma}},-\frac{\sqrt{h_{2}}}{\sqrt{\gamma}}\right) & \text { if } \quad \gamma P_{J} \geq h_{1} P_{1}+h_{2} P_{2} \\
\left(-\rho \sqrt{h_{1}},-\rho \sqrt{h_{2}}\right) & \text { if } \quad \gamma P_{J}<h_{1} P_{1}+h_{2} P_{2}
\end{array}\right.
$$

where

$$
\rho=\min \left\{\sqrt{\frac{P_{J}}{h_{1} P_{1}+h_{2} P_{2}}}, \frac{\gamma P_{J}+\sigma_{N}^{2}}{\sqrt{\gamma}\left(h_{1} P_{1}+h_{2} P_{2}\right)}\right\}
$$

and the jammer transmits as in (5). We observe that the amount of power the jammer allocates for jamming each user is proportional to that user's effective received power which is $h_{i} P_{i}$ for user $i, i=1,2$.

\section{JAMMING WITH EAVESDROPPING INFORMATION}

Now suppose that the jammer gains information about the user signals through an eavesdropping channel,

$$
Y_{e}=\sqrt{g_{1}} X_{1}+\sqrt{g_{2}} X_{2}+N_{e}
$$

We define linear jamming as

$$
J=\rho Y_{e}+N_{J}
$$

Here, we will prove that in the eavesdropping case as well, linear jamming and Gaussian signalling is a game solution. The proof of the optimality of Gaussian signalling, when the jammer is linear is similar to the previous section [4]. However, when it comes to showing the optimality of linear jamming when the users employ Gaussian signalling, the method of the previous section cannot be used, since from (18) and (19), the values of $E\left[X_{1} J\right]$ and $E\left[X_{2} J\right]$ that are achievable through linear jamming, should further satisfy

$$
\frac{E\left[X_{1} J\right]}{\sqrt{g_{1}}}=\frac{E\left[X_{2} J\right]}{\sqrt{g_{2}}}
$$

Therefore, linear jamming may not achieve all $|\boldsymbol{\Lambda}|$ values in (10) that are allowed under the power constraints. Here, we show the optimality of linear jamming, by setting up an equivalent channel. Define random variables $Z_{1}$ and $Z_{2}$ in terms of $X_{1}$ and $X_{2}$ as

$$
\begin{aligned}
& Z_{1}=X_{1}+\frac{\sqrt{g_{2}}}{\sqrt{g_{1}}} X_{2} \\
& Z_{2}=-\frac{\sqrt{g_{1} g_{2}} P_{2}}{g_{1} P_{1}+g_{2} P_{2}} X_{1}+\frac{g_{1} P_{1}}{g_{1} P_{1}+g_{2} P_{2}} X_{2}
\end{aligned}
$$

It is straightforward to verify that $Z_{1}$ and $Z_{2}$ are uncorrelated, and hence, independent Gaussian random variables. Also

$$
I\left(X_{1}, X_{2} ; Y\right)=I\left(Z_{1}, Z_{2} ; Y\right)
$$


Therefore, the game's objective function can be replaced with $I\left(Z_{1}, Z_{2} ; Y\right)$. Now, using (1), (21) and (22),

$$
Y=u_{1} Z_{1}+u_{2} Z_{2}+\sqrt{\gamma} J+N
$$

where $u_{i}$ can be expressed in terms of $h_{i}, g_{i}$ and $P_{i}, i=$ 1,2. Using (18), (21) and (22),

$$
Y_{e}=\sqrt{g_{1}} Z_{1}+N_{e}
$$

Note that $Y_{e}$ is independent of $Z_{2}$. Equations (24) and (25) define a two user, one jammer system, depicted in Figure 2, where the jammer has eavesdropping information only about one of the users. Now, the equivalent input/output mutual information is

$$
I\left(Z_{1}, Z_{2} ; Y\right)=h\left(Z_{1}, Z_{2}\right)-h\left(Z_{1}, Z_{2} \mid Y\right)
$$

The jammer can only affect the second term above,

$$
\begin{aligned}
h\left(Z_{1}, Z_{2} \mid Y\right) & =h\left(Z_{1}-a_{1} Y, Z_{2}-a_{2} Y \mid Y\right) \\
& \leq h\left(Z_{1}-a_{1} Y, Z_{2}-a_{2} Y\right) \\
& \leq \frac{1}{2} \log (1+|\boldsymbol{\Sigma}|)
\end{aligned}
$$

where $\boldsymbol{\Sigma}$ is the covariance matrix of $Z_{1}-a_{1} Y$ and $Z_{2}-a_{2} Y$. Following steps similar to those in the previous section, when the users are Gaussian, employing linear jamming together with a good choice of $a_{1}$ and $a_{2}$ can make both inequalities hold with equality, and $|\boldsymbol{\Sigma}|$ will only be a function of $E\left[Z_{1} J\right]$ and $E\left[Z_{2} J\right]$. However, $E\left[Z_{2} J\right]=0$ and therefore, $|\boldsymbol{\Sigma}|$ is only a function of $E\left[Z_{1} J\right]$. In the sequel, we show that all $E\left[Z_{1} J\right]$ values that are achievable by all feasible jamming strategies, are also achievable by some feasible linear jamming, and therefore, linear jamming achieves the largest possible upper bound in (29) and is optimal.

Using (25), the linear least squared error (LLSE) estimate of $Z_{1}$ from $Y_{e}$ is [7]

$$
\tilde{Z}_{1}\left(Y_{e}\right)=\frac{\sqrt{g_{1}} E\left[Z_{1}^{2}\right]}{\sigma_{N_{e}}^{2}+g_{1} E\left[Z_{1}^{2}\right]} Y_{e}
$$

Since $Z_{1}$ and $N_{e}$ are Gaussian, this estimate is also the minimum mean squared error (MMSE) estimate of $Z_{1}$, therefore, any other estimate of $Z_{1}$ results in a higher mean squared error. Consider any jamming signal $J$. The LLSE estimate of $Z_{1}$ from $J$ is

$$
\hat{Z}_{1}=\frac{E\left[Z_{1} J\right]}{E\left[J^{2}\right]} J
$$

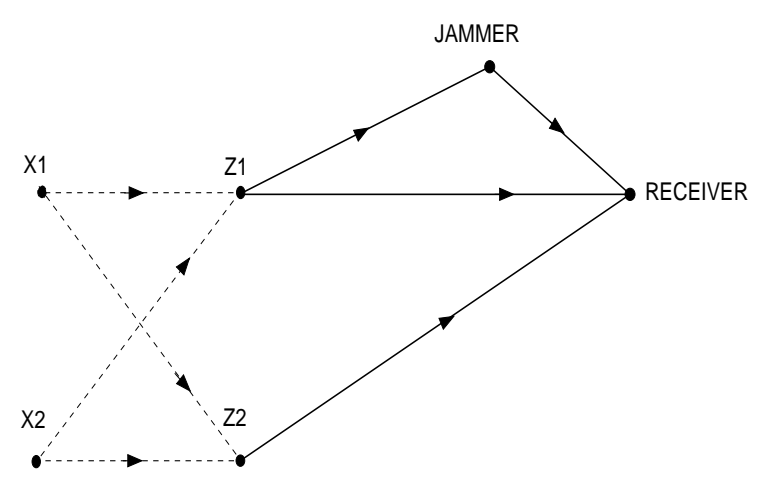

Figure 2: An interpretation of a communication system with two users and one jammer with eavesdropping information.

This is also another estimator of $Z_{1}$ from $Y_{e}$, hence the mean squared error for $\hat{Z}_{1}$ is greater than or equal to the mean squared error for $\tilde{Z}_{1}$, resulting in

$$
E^{2}\left[Z_{1} J\right] \leq \frac{g_{1} E^{2}\left[Z_{1}^{2}\right] P_{J}}{\sigma_{N_{e}}^{2}+g_{1} E\left[Z_{1}^{2}\right]}
$$

Meanwhile, using (25), all $E^{2}\left[Z_{1} J\right]$ values smaller than the right hand side of (32) are achievable by linear jamming, which means that linear jamming can achieve all feasible $|\boldsymbol{\Sigma}|$ values in (29), and this concludes the proof.

To find the optimal jamming coefficient, using (18) and (19), we write the jamming signal as

$$
J=\rho\left(\sqrt{g_{1}} X_{1}+\sqrt{g_{2}} X_{2}+N_{e}\right)+N_{J}
$$

and the jammer's optimization problem is

$$
\begin{aligned}
\min _{\left\{\rho, \sigma_{N_{J}}^{2}\right\}} & \frac{\left(\sqrt{h_{1}}+\rho \sqrt{\gamma g_{1}}\right)^{2} P_{1}+\left(\sqrt{h_{2}}+\rho \sqrt{\gamma g_{2}}\right)^{2} P_{2}}{\gamma \rho^{2} \sigma_{N_{e}}^{2}+\gamma \sigma_{N_{J}}^{2}+\sigma_{N}^{2}} \\
\text { s.t. } & \rho^{2}\left(g_{1} P_{1}+g_{2} P_{2}+\sigma_{N_{e}}^{2}\right)+\sigma_{N_{J}}^{2} \leq P_{J}
\end{aligned}
$$

The KKTs for this problem can be solved using numerical optimization [4].

\section{JAMMING IN FADING MULTI-USER AWGN CHANNELS}

In the rest of the paper, we investigate the optimum user/jammer strategies when the channels are fading. We use the term CSI, for the channel state information on the links from the users to the receiver, and assume that the link between the jammer and the receiver is non-fading. The receiver is assumed to know the CSI, while various assumptions are made on the availability of the CSI at the jammer. 


\section{NO CSI AT THE TRANSMITTERS}

When the transmitters do not have the CSI, it is reasonable to assume that the jammer does not have the CSI either. In the sequel, we show that the jammer's information about the transmitted signals will be irrelevant and therefore, it will not make any difference whether it has perfect or noisy information about the transmitted signals. This is a multi-user generalization of the results of [2] in a SISO system.

Assuming that the user links are fading and the jammer link is non-fading, the received signal is

$$
Y=H_{1} X_{1}+H_{2} X_{2}+\sqrt{\gamma} J+N
$$

Similar arguments as in the case of correlated jamming in non-fading channels can be used to show that the strategies corresponding to the game solution will be Gaussian signalling and linear jamming [4]. The jamming signal is as in (5) and the jammer minimizes

$$
E\left[\log \left(1+\frac{\left|H_{1}+\sqrt{\gamma} \rho_{1}\right|^{2} P_{1}+\left|H_{2}+\sqrt{\gamma} \rho_{2}\right|^{2} P_{2}}{\gamma \sigma_{N_{J}}^{2}+\sigma_{N}^{2}}\right)\right]
$$

subject to $\rho_{1}^{2} P_{1}+\rho_{2}^{2} P_{2}+\sigma_{N_{J}}^{2} \leq P_{J}$. The random variables $H_{1}$ and $H_{2}$ are circularly symmetric complex Gaussian with zero mean, therefore, the optimal jamming coefficients are $\rho_{1}=\rho_{2}=0$ and the jammer disregards its information about the user signals [4].

\section{UNCORRELATED JAMMING WITH CSI AT THE TRANSMITTERS}

We now consider a two user fading channel with a jammer who does not have any information about the user signals. We assume that the state of the user links are known to the users. Capacity of fading channels with CSI both at the transmitter and the receiver when there is no jammer, has been investigated in [8] and [9], and optimum user strategies have been derived. We consider the same problem when there is a jammer in the system. First consider the single-user case

$$
Y=H X+\sqrt{\gamma} J+N
$$

When the CSI is available both at the transmitter and the receiver, the input/output mutual information is

$$
C=I(X ; Y \mid H)
$$

For $\left(X_{1}, X_{2}, Y \mid H\right) \sim f\left(x_{1} \mid h\right) f\left(x_{2} \mid h\right) f\left(y \mid x_{1}, x_{2}, h\right), C$ is a convex function of $f(y \mid x, h)$ for any $f(x \mid h)$, and a con- cave function of $f(x \mid h)$ for any fixed $f(y \mid x, h)$. The mutual information sub-game for any given channel state has a Nash equilibrium solution which is Gaussian signalling for the user and linear jamming for the jammer. If in addition, a Nash equilibrium solution exists over all feasible power allocation strategies of the user and the jammer, that solution along with the signalling and jamming strategies specified as the solution of the subgames at each channel state, will give the overall game solution. We proceed with first assuming that while the user has the CSI, the jammer does not have the CSI, and then assuming that the CSI is available both at the user and at the jammer.

If the jammer has no information about the fading channel state, the best strategy for the jammer is to transmit Gaussian noise and the capacity is

$$
C=\frac{1}{2} E\left[\log \left(1+\frac{h P(h)}{\sigma_{N}^{2}+\gamma P_{J}}\right)\right]
$$

where $P(h)$ is the user power at fading level $h$ which should satisfy

$$
E[P(h)] \leq P
$$

The best user power allocation is waterfilling over the equivalent parallel AWGN channels [8], i.e.,

$$
P(h)=\left(\frac{1}{\lambda}-\frac{\sigma_{N}^{2}+\gamma P_{J}}{h}\right)^{+}
$$

where $(x)^{+}=x$ if $x \geq 0$, and 0 otherwise, and $\lambda$ is a constant chosen to enforce the user power constraint. The corresponding two user system, where the jammer is not aware of the user channel coefficients, is a straightforward extension of the results in [10] where only one user transmits at a time. The jammer will again use all its power to add Gaussian noise.

Next, we assume that the uncorrelated jammer has full CSI. At each channel state, the jammer transmits Gaussian noise at the power level allocated to that state and the capacity is

$$
C=\frac{1}{2} E\left[\log \left(1+\frac{h P(h)}{\sigma_{N}^{2}+\gamma J(h)}\right)\right]
$$

where $J(h)$ is the jammer power at fading level $h$. The user power constraint is the same as (39), and the jammer power constraint is

$$
E[J(h)] \leq P_{J}
$$


The capacity is a concave function of $P$ for fixed $J$ and a convex function of $J$ for fixed $P$, therefore, the mutual information game has a solution [4]. The KKTs for each state-allocated user and jammer power result in [4]

$$
P(h)=\left\{\begin{array}{lll}
\left(\frac{1}{\lambda}-\frac{\gamma \sigma_{N}^{2}}{h}\right)^{+} & \text {if } \quad h<\frac{\sigma_{N}^{2} \gamma \lambda}{\gamma-\sigma_{N}^{2} \mu} \\
\frac{h}{\lambda\left(h+\gamma \frac{\lambda}{\mu}\right)} & \text { if } \quad h \geq \frac{\sigma_{N}^{2} \gamma \lambda}{\gamma-\sigma_{N}^{2} \mu}
\end{array}\right.
$$

and

$$
J(h)= \begin{cases}0 & \text { if } \quad h<\frac{\sigma_{N}^{2} \gamma \lambda}{\gamma-\sigma_{N}^{2} \mu} \\ \frac{h}{\mu\left(h+\gamma \frac{\lambda}{\mu}\right)}-\frac{\sigma_{N}^{2}}{\gamma} & \text { if } \quad h \geq \frac{\sigma_{N}^{2} \gamma \lambda}{\gamma-\sigma_{N}^{2} \mu}\end{cases}
$$

where $\lambda$ and $\mu$ are found using the user and jammer power constraints.

Following similar arguments as in the two user system without a jammer in [10], the results can be extended to a two user system where the jammer is uncorrelated but it has access to the CSI. The power allocation strategies will be functions of both channel states $h_{1}$ and $h_{2}$, and at any given pair of $h_{1}$ and $h_{2}$, only the user with a relatively better channel state transmits [4].

\section{CORRELATED JAMMING WITH CSI AT THE TRANSMITTERS}

In this section, we consider a two user fading channel with a jammer who knows the user signals. We assume that the user links are fading and the state of the user links are known to the users and the correlated jammer. We first show that this game does not always have a Nash equilibrium solution, and then, find the max-min user strategies and the corresponding jamming strategy. The input/output mutual information is as in (37). The user and jammer power constraints can be written as

$$
\begin{aligned}
E\left[E\left[X^{2} \mid H\right]\right] & \leq P \\
E\left[E\left[J^{2} \mid H\right]\right] & \leq P_{J}
\end{aligned}
$$

where $E\left[X^{2} \mid H=h\right]$ and $E\left[J^{2} \mid H=h\right]$ are the user and jammer powers allocated to the fading level $H=h$. Any pair of user and jammer strategies, results in a pair of user and jammer power allocations. Therefore, the game's solution can be described as a pair of user and jammer power allocations, along with the user and jammer signalling functions at each channel state. Using our results for the non-fading channels, we have that irrespective of the existence or non-existence of optimal power allocation functions for the user and the jammer, the sub-games at each channel state, always have a solution which is Gaussian signalling for the users and linear jamming for the jammer.

Since the jammer knows the channel state and the transmitted signal, the received signal is

$$
Y=(\sqrt{h}+\rho(h)) X+N_{J}+N
$$

where the variance of $N_{J}$ is also a function of $h, \sigma_{N_{J}}^{2}(h)$. Given a pair of power allocation functions $P(h)$ and $J(h)$, the capacity is

$$
C=\frac{1}{2} E\left[\log \left(1+\frac{(\sqrt{h}+\rho(h))^{2} P(h)}{\sigma_{N}^{2}+\sigma_{N_{J}}^{2}(h)}\right)\right]
$$

where $\rho(h)$ and $\sigma_{N_{J}}^{2}(h)$ describe the optimal linear jamming for an equivalent non-fading channel with attenuation $h$, and user and jammer powers $P(h)$ and $J(h)$. The power constraints of the user and the jammer are as in (39) and (42). The capacity here does not have the convexity/concavity properties. In the sequel, we show that a pair of strategies, which is simultaneously optimal for the user and the jammer, does not always exist. We first assume that the user chooses its strategy once at the beginning of the communication, knowing that the jammer will employ the corresponding optimal jamming strategy. We then characterize the user and jammer strategies in this scenario. If the game had a solution, it would have been this pair of user and jammer strategies, however we prove the converse.

Given any user power allocation function $P(h)$, we find the jammer's best response, where a best response describes what a player does, given the other player's move [6]. In this case, a best response is the jammer's best power allocation, given a fixed user power allocation. The jammer's best response can also be thought of as a pair of functions $\rho(h)$ and $\sigma_{N_{J}}^{2}(h)$ which minimizes

$$
C=\frac{1}{2} E\left[\log \left(1+\frac{(\sqrt{h}+\rho(h))^{2} P(h)}{\sigma_{N}^{2}+\sigma_{N_{J}}^{2}(h)}\right)\right]
$$

and $\rho(h)$ and $\sigma_{N_{J}}^{2}(h)$ are constrained such that

$$
E\left[\rho^{2}(h) P(h)+\sigma_{N_{J}}^{2}(h)\right] \leq P_{J}
$$

Assuming that $\sigma_{N}^{2}=0$, the jammer's strategy should always be such that whenever the user transmits, $\sigma_{N_{J}}^{2}(h)>0$, since otherwise, the capacity would be in- 
finite. The first order KKT conditions for the optimal jamming strategy result in

$$
\begin{aligned}
& \left(\rho(h), \sigma_{N_{J}}^{2}(h)\right)= \\
& \left\{\begin{array}{lll}
(-\sqrt{h}, 0) & \text { if } & h P(h) \leq \frac{1}{\lambda} \\
\left(-\frac{1}{\lambda \sqrt{h} P(h)}, \frac{1}{\lambda}-\frac{1}{\lambda^{2} h P(h)}\right) & \text { if } & h P(h)>\frac{1}{\lambda}
\end{array}\right.
\end{aligned}
$$

where $\lambda$ is chosen to satisfy the jammer's power constraint. The total power that the jammer allocates to each channel state is found as

$$
J(h)= \begin{cases}h P(h) & \text { if } \quad h P(h) \leq \frac{1}{\lambda} \\ \frac{1}{\lambda} & \text { if } \quad h P(h)>\frac{1}{\lambda}\end{cases}
$$

which describes the best response of the jammer, to the user power allocation $P(h)$.

We now derive the max-min user power allocation. First note that the function inside the expectation in (49) is zero for $h P(h) \leq 1 / \lambda$, therefore, in the optimal user power allocation, $P(h)$ is either zero, or such that $h P(h)>1 / \lambda$. The capacity can now be written as

$$
C=\frac{1}{2} E[\log (\lambda \sqrt{h} P(h))]
$$

The KKT condition for the user power, whenever the user transmits, results in $P(h)=\frac{1}{\mu}$, for which

$$
J(h)=\left\{\begin{array}{lll}
0 & \text { if } \quad h \leq \frac{\mu}{\lambda} \\
\frac{1}{\lambda} & \text { if } \quad h>\frac{\mu}{\lambda}
\end{array}\right.
$$

These user and jammer power allocations are illustrated in Figure 3 where $\mu$ and $\lambda$ are chosen to satisfy the user and jammer power constraints.

We now show that when the jammer employs (54), the current user strategy is not optimal. Consider two fading levels $u^{+}>\mu / \lambda$ and $u^{-}<\mu / \lambda$ close enough to $h=\mu / \lambda$ such that

$$
u^{-} \simeq u^{+} \simeq \mu / \lambda
$$

We have $J\left(u^{-}\right)=0$ and $J\left(u^{+}\right)=1 / \lambda$. Obviously, it is not optimal for the user to transmit at $u^{+}$while not transmitting at $u^{-}$, therefore, the pair of the user and jammer power allocations derived (which corresponds to the user max-min solution), is not a game solution, and the game does not admit a solution.

Even though the max-min solution derived above is not the game Nash equilibrium solution, it is the optimal pair of user and jammer strategies in a system where a conservative user would like to guarantee itself with

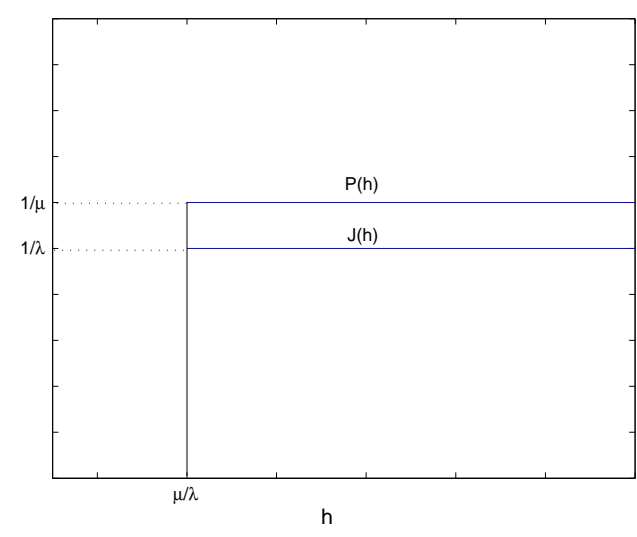

Figure 3: Max-min user power allocation and the corresponding jammer best response power allocation.

some capacity value. It also describes the best strategy for a user which is less dynamic than the jammer in terms of changing the transmission strategy, and can choose its strategy only once.

\section{REFERENCES}

[1] M. Médard, "Capacity of Correlated Jamming Channels," Allerton, 1997.

[2] A. Kashyap, T. Basar and R. Srikant, "Correlated jamming on MIMO Gaussian fading channels," IEEE Tran. on Inf. The., Sep. 2004.

[3] A. Bayesteh, M. Ansari and A. K. Khandani, "Effect of Jamming on the Capacity of MIMO Channels," Allerton, 2004.

[4] S. Shafiee and S. Ulukus, "Correlated Jamming in Multiple Access Channels," CISS, 2005.

[5] T. M. Cover and J. A. Thomas, Elements of Information Theory, John Wiely \& Sons, 1991.

[6] D. Fudenberg and J. Tirole, Game Theory, MIT Press, 1991.

[7] H. V. Poor, An Introduction to Signal Detection and Estimation, 2nd Ed., Springer-Verlag, 1994.

[8] A. Goldsmith and P. Varaiya, "Capacity of fading channels with channel side information," IEEE Tran. on Inf. The., Nov. 1997.

[9] G. Caire and S. Shamai, "On the Capacity of Some Channels with Channel State Information," IEEE Tran. on Inf. The., Sep. 1999.

[10] R. Knopp and P. A. Humblet, "Information capacity and power control in single-cell multiuser communications," IEEE ICC, Jun. 1995. 